\title{
Implications of Incidental Germline Findings Identified In the Context of Clinical Whole Exome Sequencing for Guiding Cancer Therapy
}

Bryan P. Schneider, MD ${ }^{1,2}$; Leigh Anne Stout, MS ${ }^{1,2}$; Santosh Philips, PhD $^{1}$; Courtney Schroeder, MS ${ }^{1,2}$; Susanna F. Scott, MPA ${ }^{1,2}$; Cynthia Hunter, MS ${ }^{1,2}$; Nawal Kassem, MD ${ }^{1,2}$; Patrick J. Kiel, PharmD ${ }^{1,2}$; and Milan Radovich, PhD ${ }^{1,2}$

PURPOSE Identification of incidental germline mutations in the context of next-generation sequencing is an unintended consequence of advancing technologies. These data are critical for family members to understand disease risks and take action.

PATIENTS AND METHODS A retrospective cohort analysis was conducted of 1,028 adult patients with metastatic cancer who were sequenced with tumor and germline whole exome sequencing (WES). Germline variant call files were mined for pathogenic/likely pathogenic (P/LP) variants using the ClinVar database and narrowed to high-quality submitters.

RESULTS Median age was 59 years, with $16 \%$ of patients $\leq 45$ years old. The most common tumor types were breast cancer (12.5\%), colorectal cancer (11.5\%), sarcoma (9.3\%), prostate cancer (8.4\%), and lung cancer (6.6\%). We identified 3,427 P/LP variants in 471 genes, and $84 \%$ of patients harbored one or more variant. One hundred thirty-two patients (12.8\%) carried a P/LP variant in a cancer predisposition gene, with BRCA2 being the most common (1.6\%). Patients with breast cancer were most likely to carry a P/LP variant $(19.2 \%)$. One hundred ten patients (10.7\%) carried a P/LP variant in a gene that would be recommended by the American College of Medical Genetics and Genomics to be reported as a result of clinical actionability, with the most common being ATP7B (2.7\%), BRCA2 (1.6\%), MUTYH (1.4\%), and BRCA1 (1\%). Of patients who carried a P/LP variant in a cancer predisposition gene, only $53 \%$ would have been offered correct testing based on current clinical practice guidelines. Of 471 mutated genes, 231 genes had a P/LP variant identified in one patient, demonstrating significant genetic heterogeneity.

CONCLUSION The majority of patients undergoing clinical cancer WES harbor a pathogenic germline variation. Identification of clinically actionable germline findings will create additional burden on oncology clinics as broader WES becomes common.

JCO Precis Oncol 4:1109 1121. @ 2020 by American Society of Clinical Oncology

\section{INTRODUCTION}

Next-generation sequencing (NGS) of a patient's tumor has become a common approach to identify targetable genomic aberrations in patients with a variety of advanced malignancies. Currently, the US Food and Drug Administration has approved at least one panel-based test ${ }^{1}$ and, more recently, a comprehensive NGS test with coverage of the whole exome, and more than three quarters of oncologists nationwide use some type of NGS-based test in routine practice. ${ }^{2}$ As the number of novel drug targets increases and the cost of sequencing decreases, ${ }^{3}$ comprehensive testing will become more cost efficient and more commonly used in the community setting.

One of the unintended consequences of conducting tumor NGS is the incidental identification of pathogenic germline variants. ${ }^{4-9}$ For testing that does not include paired germline DNA, the detection of a mutation in a loci known to be consistent with a pathogenic germline mutation must prompt, at the very least, consideration of confirmatory germline testing. For most comprehensive, whole exome-based tests, paired germline is necessary to filter out the vast number of variants tested. In any case, the detection of concerning germline findings is complicated by multiple factors, including the complicated and dynamic characterization of variant pathogenicity (which is obviously designed for determining the targetability of a somatic variant) and the clinical actionability of the various pathogenic variants. The technology to identify such variants exceeds our ability to accurately determine pathogenicity of variants. It is not uncommon for laboratories to have discordant classifications of variants depending on their interpretation of the 


\section{CONTEXT}

\section{Key Objective}

What are some of the commonly identified germline findings when whole exome sequencing (WES) is performed in an oncology clinic with the intent of guiding cancer therapy?

\section{Knowledge Generated}

Patients undergoing WES are often found to carry at least one germline pathogenic variant, adding to the complexity of their care. Pathogenic variants were most commonly identified in non-cancer predisposition genes; however, $12.8 \%$ of patients carried a pathogenic variant in a cancer predisposition gene.

\section{Relevance}

As paired germline and tumor analysis becomes more common in oncology clinics, careful approaches to pretest counseling and result disclosure are critical. A multidisciplinary approach, including clinical genetics, is necessary to provide optimal care for both patients and their family members.

data. ${ }^{10-12}$ These classifications may or may not be routinely updated by laboratories as new data emerge. Furthermore, a particular variant's pathogenicity may be dependent on whether it is somatic or germline in origin.

Equally important is the complex clinical context of such findings given that most patients have a life-limiting malignancy, receive the test without optimal pretest counseling, and have agreed to the test for a completely different reason (ie, to identify a drug target). Currently, ASCO supports the communication of medically relevant and incidental germline findings to patients, ${ }^{13}$ and the vast majority of patients agree to receive these findings when given the option to consent. ${ }^{14,15}$ Multiple studies have found that the occurrence of germline cancer predisposition variants is not rare in this population, ranging from $3.0 \%$ to $12.6 \%{ }^{4-9}$ However, these data from panel-based testing are not comprehensive and lack risk-specific details such as family history. The largest study to date from The Cancer Genome Atlas (TCGA; $n=10,398$ ) reported a frequency of $8 \%{ }^{16}$ but only included cancer-specific variants and did not have companion clinical annotation. Here, we present, to our knowledge, the first expansive germline findings for patients with cancer receiving comprehensive whole exome sequencing (WES) of the tumor and matched germline with annotated history with the primary intent to identify drug targets.

\section{PATIENTS AND METHODS}

\section{Patients}

Our retrospective analysis included 1,028 consecutive adult patients with any histologic type of metastatic cancer, who were referred to the Indiana University Health Precision Genomics clinic between January 12, 2016, and March 31, 2019, and who underwent paired tumor and germline WES. The primary intent for testing was to guide cancer therapy. Each patient had adequate tumor DNA to meet the minimum standards for WES. All patients also submitted a blood sample or buccal swab for germline analysis. This study was approved by the Indiana University Institutional Review Board.

\section{Molecular Analysis of Patient Samples}

DNA samples were obtained from each patient and sent to NantOmics (Culver City, CA) for paired germline/somatic testing. Clinical Laboratory Improvement Amendments (CLIA)-based somatic WES was performed on each sample.

In addition, exome sequencing of germline DNA was performed with CLIA reporting of the American College of Medical Genetics and Genomics (ACMG) cancer predisposition genes. DNA sequencing libraries were prepared from normal blood or buccal samples using the KAPA HyperPrep kit (Roche, Indianapolis, IN) and sequenced on an Illumina Sequencing Platform (Illumina, San Diego, CA). DNA sequencing data were aligned to the human genome (hg19) using the Burrows-Wheeler Aligner algorithm. Duplicated reads were marked by samblaster, and indel realignment and base quality recalibration were performed using GATK v2.3. Each variant was sequenced to a minimum depth of 10 reads and had a minimum alternate allele fraction of 0.25 in the normal sequencing data. Variant call format (VCF) files containing germline variants were generated. NantOmics WES CLIA sequencing has demonstrated $>95 \%$ sensitivity and $>99 \%$ specificity for germline single nucleotide polymorphisms and germline insertions and deletions.

\section{Variant Interpretation for Hereditary Disease}

Variants from germline VCF files were then annotated with ClinVar pathogenicity classifications using the Golden Helix SVS 8.8.3 software (Golden Helix, Bozeman, MT). Variants were filtered for those that were classified as either pathogenic or likely pathogenic (P/LP) by any one of the following ClinVar submitters: Ambry Genetics (Aliso Viejo, CA), Invitae (San Francisco, CA), or GeneDx (Gaithersburg, $M D$ ). These laboratories were selected because of their robust testing menu, thorough variant interpretation 
processes, and frequent submissions and updates to ClinVar. All genes were included in this analysis regardless of disease category. Known artifacts of the assay were removed for quality control.

\section{RESULTS}

\section{Patient Characteristics}

One thousand twenty-eight patients who underwent paired tumor and germline WES were included in this analysis. The patient characteristics are listed in Table 1 . The median age was 59 years, with $16 \%$ of patients $<45$ years of age and $54 \%<60$ years of age. Eighty-six percent of the population self-defined their race as White or Caucasian

TABLE 1. Patient Characteristics and Tumor Types

\section{Characteristics}

\begin{tabular}{|c|c|}
\hline \multicolumn{2}{|l|}{ Age, years } \\
\hline$\leq 45$ & $164(16)$ \\
\hline 4660 & $390(38)$ \\
\hline 6175 & $401(39)$ \\
\hline$\geq 76$ & $73(7)$ \\
\hline \multicolumn{2}{|l|}{ Sex } \\
\hline Female & $542(53)$ \\
\hline Male & $486(47)$ \\
\hline \multicolumn{2}{|l|}{ Race } \\
\hline White or Caucasian & $890(87)$ \\
\hline Black or African American & $84(8)$ \\
\hline Unknown & $34(3)$ \\
\hline Asian & $17(2)$ \\
\hline American Indian & $3(0.3)$ \\
\hline \multicolumn{2}{|l|}{ Tumor type } \\
\hline Breast & $146(13)$ \\
\hline Colorectal & $134(12)$ \\
\hline Sarcoma & $109(9)$ \\
\hline Prostate & $98(8)$ \\
\hline Lung & $77(7)$ \\
\hline Head and neck & $59(5)$ \\
\hline CNS tumor & $52(5)$ \\
\hline Ovarian & $58(5)$ \\
\hline Pancreas & $47(4)$ \\
\hline Melanoma & $44(4)$ \\
\hline Urothelial & $42(4)$ \\
\hline Renal & $30(3)$ \\
\hline Cholangiocarcinoma & $26(2)$ \\
\hline Thymoma & $24(2)$ \\
\hline Endometrial & $23(2)$ \\
\hline Thyroid & $23(2)$ \\
\hline Unknown primary & $18(2)$ \\
\hline Other & 157 (14) \\
\hline
\end{tabular}

and $8 \%$ as Black or African American. The most common tumor types were breast cancer (12.5\%), colorectal cancer (11.5\%), sarcoma $(9.3 \%)$, prostate cancer $(8.4 \%)$, and lung cancer (6.6\%).

\section{Genomic Landscape}

A total of 3,427 P/LP germline variants were identified. Of these, there were 855 unique P/LP variants in a total of 471 genes. The frequency and breakdown of P/LP variants is summarized in Figure 1. Eight hundred sixty-two (84\%) of 1,028 patients carried a P/LP variant of any type (cancer or noncancer) in any gene (ACMG- or non-ACMG-designated variant; Data Supplement). Of 471 mutated genes, a single $\mathrm{P} / \mathrm{LP}$ variant was identified in 231 genes $(49 \%)$, demonstrating significant genetic heterogeneity. The most commonly mutated genes (frequency $>1.5 \%$ ) are listed in Table 2, with HFE being the most common (37.4\%) followed by MCR1 $(33.7 \%)$ and GALT $(17 \%)$. The vast majority of these 29 genes have an autosomal recessive inheritance pattern, and only two (6.9\%) of 29 are recommended by the ACMG to have results returned to the patient.

\section{Frequency of Cancer Predisposition Genes, Concordance, and Predictive Capacity of the Variant}

A total of $12.8 \%$ of patients carried a cancer predisposition variant (Table 3 ). The most commonly mutated cancer predisposition genes were BRCA2 (1.6\%), CHEK2 (1.4\%), MUTYH (1.4\%), ATM (1.0\%), and BRCA1 (1.0\%). Concordance between genes and tumor types was determined by a group of licensed genetic counselors using wellestablished, widely accepted cancer risks based on an

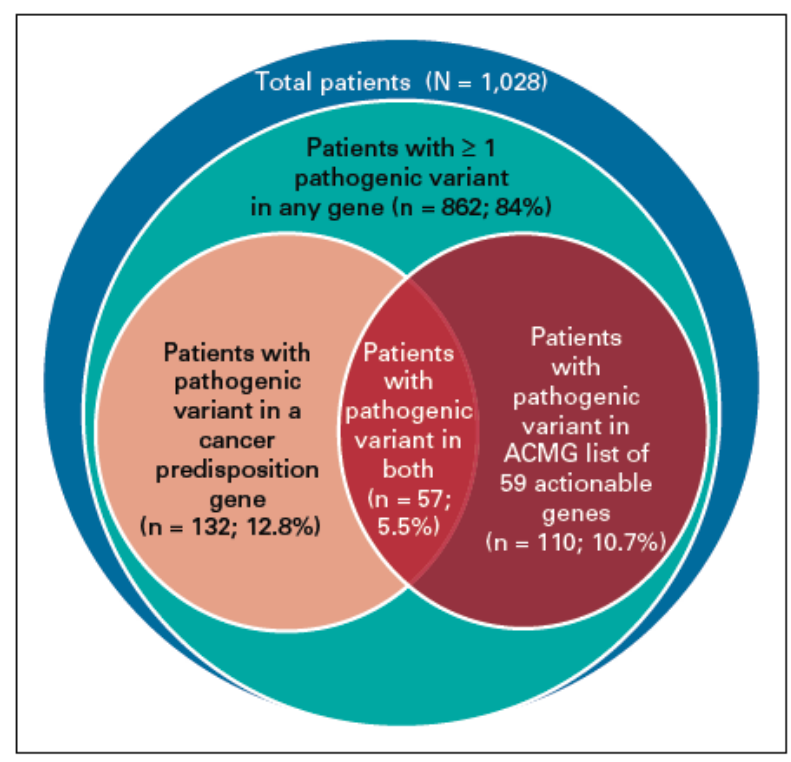

FIG 1. Pathogenic variants in 1,028 patients with advanced cancer who underwent paired tumor and germline whole exome se quencing. ACMG, American College of Medical Genetics and Genomics. 
TABLE 2. Genes Mutated in $\geq 1.5 \%$ of Our Patient Cohort

\begin{tabular}{|c|c|c|c|c|}
\hline Gene & Associated Condition & Inheritance Pattern & $\begin{array}{l}\text { Included in ACMG List of } 59 \\
\text { Actionable Genes }\end{array}$ & $\begin{array}{l}\text { No. of Patients Carrying } \\
\text { PLP Variant (\%) }\end{array}$ \\
\hline$M C 1 R$ & $\begin{array}{l}\text { Modifier gene for hair and skin color; } \\
\text { possible increased risk for melanoma }\end{array}$ & $\begin{array}{l}\text { Modifier; possible autosomal dominant } \\
\text { melanoma risk for some variants }\end{array}$ & No & $346(33.7)$ \\
\hline GALT & Galactosemia & Autosomal recessive & No & $175(17.0)$ \\
\hline CBS & Homocystinuria & Autosomal recessive & No & $170(16.5)$ \\
\hline SERPIN1A & $\alpha_{1}$ Antitrypsin & Autosomal codominant & No & 115 (11.2) \\
\hline BTD & Biotinidase deficiency & Autosomal recessive & No & $79(7.7)$ \\
\hline GJB2 & Nonsyndromic hearing loss & Autosomal recessive & No & $63(6.1)$ \\
\hline ACADS & SCAD deficiency & Autosomal recessive & No & $58(5.6)$ \\
\hline CFTR & $\begin{array}{l}\text { Cystic fibrosis and congenital absence of } \\
\text { the vas deferens }\end{array}$ & Autosomal recessive & No & $32(3.1)$ \\
\hline SPG7 & Spastic paraplegia 7 & Autosomal recessive & No & $29(2.8)$ \\
\hline ATP7B & Wilson disease & Autosomal recessive & Yes & $28(2.7)$ \\
\hline$A B C C 6$ & Pseudoxanthoma elasticum & Autosomal recessive & No & $27(2.6)$ \\
\hline MYO6 & $\begin{array}{l}\text { Deafness, autosomal dominant } 22 \\
\text { deafness, autosomal recessive } 37\end{array}$ & $\begin{array}{l}\text { Autosomal dominant/autosomal } \\
\text { recessive }\end{array}$ & No & $24(2.3)$ \\
\hline MPO & Myeloperoxidase deficiency & Autosomal recessive & No & $24(2.3)$ \\
\hline$A C A D M$ & $\begin{array}{l}\text { Medium chain acyl CoA dehydrogenase } \\
\text { deficiency }\end{array}$ & Autosomal recessive & No & $23(2.2)$ \\
\hline SI & Congenital sucrase isomaltase deficiency & Autosomal recessive & No & $17(1.7)$ \\
\hline$F 2$ & Prothrombin deficiency & Autosomal recessive & No & $17(1.7)$ \\
\hline DHCR7 & Smith Lemli Opitz syndrome & Autosomal recessive & No & $17(1.7)$ \\
\hline SLC26A4 & Pendred syndrome & Autosomal recessive & No & $16(1.6)$ \\
\hline BRCA2 & $\begin{array}{l}\text { Hereditary breast and ovarian cancer } \\
\text { syndrome/Fanconi anemia }\end{array}$ & $\begin{array}{l}\text { Autosomal dominant/autosomal } \\
\text { recessive }\end{array}$ & Yes & $16(1.6)$ \\
\hline PKHD1 & Polycystic kidney disease & Autosomal recessive & No & $15(1.5)$ \\
\hline
\end{tabular}

Abbreviations: ACMG, American College of Medical Genetics and Genomics; CoA, coenzyme A; P/LP, pathogenic or likely pathogenic; SCAD, short chain acyl CoA dehydrogenase; TAR, thrombocytopenia with absent radius.

accumulation of current data. After removing heterozygous MUTYH and NTHL1 carriers, only $56 \%$ of patients with a cancer predisposition variant had a tumor type concordant with the carriage of that cancer predisposition gene. Specifically, $62 \%$ of patients with a BRCA2 P/LP variant had a malignancy either previously or currently that would have been expected by carriage of a $B R C A 2 \mathrm{P} / \mathrm{LP}$ variant. Similarly, $64 \%, 30 \%$, and $90 \%$ of the CHEK2, ATM, and
BRCA1 carriers, respectively, had the tumor type either previously or currently that would have been expected by the P/LP variant. Patients with the following tumor types were most likely to carry a germline P/LP variant: breast cancer $(19.2 \%)$, prostate cancer $(18.4 \%)$, ovarian cancer (15.5\%), and cholangiocarcinoma (15.4\%); Data Supplement. Two of the $26 B R C A 1$ or BRCA2 P/LP variants identified were one of the three common Ashkenazi Jewish 
TABLE 3. Concordance of Cancer Diagnoses for Those Carrying a Cancer Predisposition Gene and Predictive Capacity of Pedigree

Cancer Predisposition

Gene

Tumor Types Seen
No. of Patients Who Would Not Have Been

No. of Tumor Types Concordant With Gene (\%)

Family History (\%)

11 (52)

\begin{tabular}{|c|c|c|c|}
\hline$B R C A 2(\mathrm{n} \quad 16)$ & 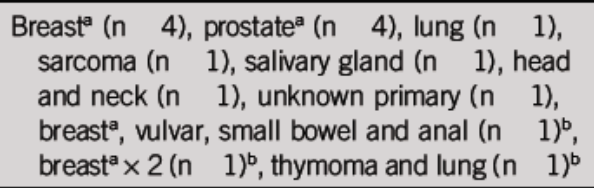 & $11(52)$ & $3(19)$ \\
\hline CHEK2 (n 14) & 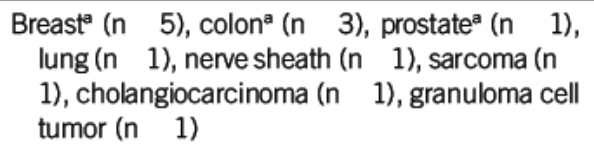 & $9(64)$ & $5(36)$ \\
\hline MUTYH (n 14) & 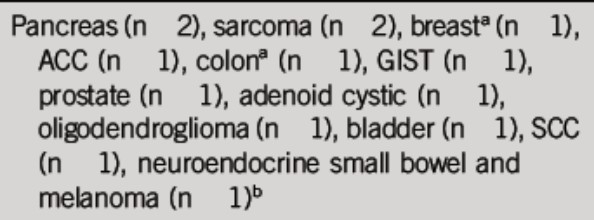 & $2(13)$ & $12(86)$ \\
\hline$B R C A 1$ (n 10) & 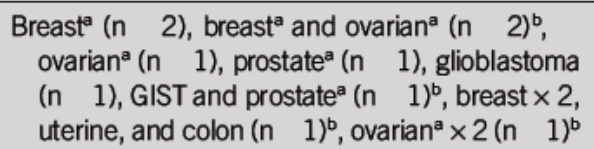 & $13(77)$ & $0(0)$ \\
\hline MITF (n 8) & $\begin{array}{l}\text { Breast (n 3), cholangiocarcinoma }\left(\begin{array}{ll}n & 2\end{array}\right) \text {, } \\
\text { pancreas }(n \quad 1) \text {, ACC }\left(\begin{array}{ll}n & 1\end{array}\right) \text {, sarcoma and } \\
\text { GIST }\left(\begin{array}{lll}n & 1)^{b}\end{array}\right.\end{array}$ & $0(0)$ & $7(88)$ \\
\hline NTHL1 (n 8$)^{c}$ & 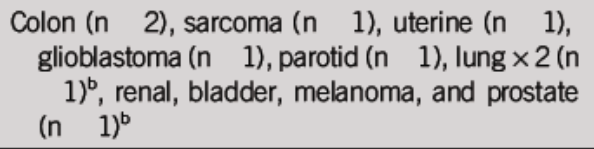 & $0(0)$ & $7(88)$ \\
\hline$N F 1(n \quad 3)$ & Sarcoma (n 2$)$, sarcoma ${ }^{a} \times 2(n \quad 1)^{b}$ & $4(100)$ & $0(0)$ \\
\hline FANCC ( $\mathrm{n} 3$ ) & $\begin{array}{l}\text { Sarcoma (n 1), ovarian (n 1), sarcoma } \times 2 \text { (n } \\
1)^{b}\end{array}$ & $0(0)$ & $3(100)$ \\
\hline$N B N\left(\begin{array}{ll}n & 3\end{array}\right)$ & Breast $^{\mathrm{a}}(\mathrm{n} \quad$ 2), bladder ( $\mathrm{n}$ 1) & $2(67)$ & $1(33)$ \\
\hline PALB2 (n 3) & Ureter ( $n \quad 1)$, ovarian ${ }^{a}(n \quad 1)$, melanoma ( $\left.n \quad 1\right)$ & $1(33)$ & $1(33)$ \\
\hline$M S H 2$ (n 2) & Colona (n 2) & $2(100)$ & $0(0)$ \\
\hline$B R I P 1(\mathrm{n} \quad 2)$ & $\begin{array}{l}\text { Cholangiocarcinoma (n } 1 \text { ), breast }{ }^{\mathrm{a}} \text { and ovarian } \\
(\mathrm{n} \quad 1)^{\mathrm{b}}\end{array}$ & $2(66)$ & $1(50)$ \\
\hline$M L H 1(\mathrm{n} \quad 2)$ & Colon $^{a}$ (n 1), gastric ${ }^{a}\left(\begin{array}{ll}n & 1\end{array}\right)$ & $2(100)$ & $0(0)$ \\
\hline MEN1 (n 2) & $\begin{array}{l}\text { Pancreas }^{a} \text { and thymic } \\
\text { pancreas }^{a}\left(\begin{array}{lll}n & 1\end{array}\right)^{b}\end{array}$ & $4(100)$ & $0(0)$ \\
\hline RECQL4 (n 2) & Gastroesophageal junction ( $(n \quad 2)$ & $0(0)$ & $2(100)$ \\
\hline$S D H B(n \quad 1)$ & Pheochromocytoma $^{a}\left(\begin{array}{ll}n & 1\end{array}\right)$ & $1(100)$ & $0(0)$ \\
\hline
\end{tabular}

(Continued on following page) 
TABLE 3. Concordance of Cancer Diagnoses for Those Carrying a Cancer Predisposition Gene and Predictive Capacity of Pedigree (Continued)

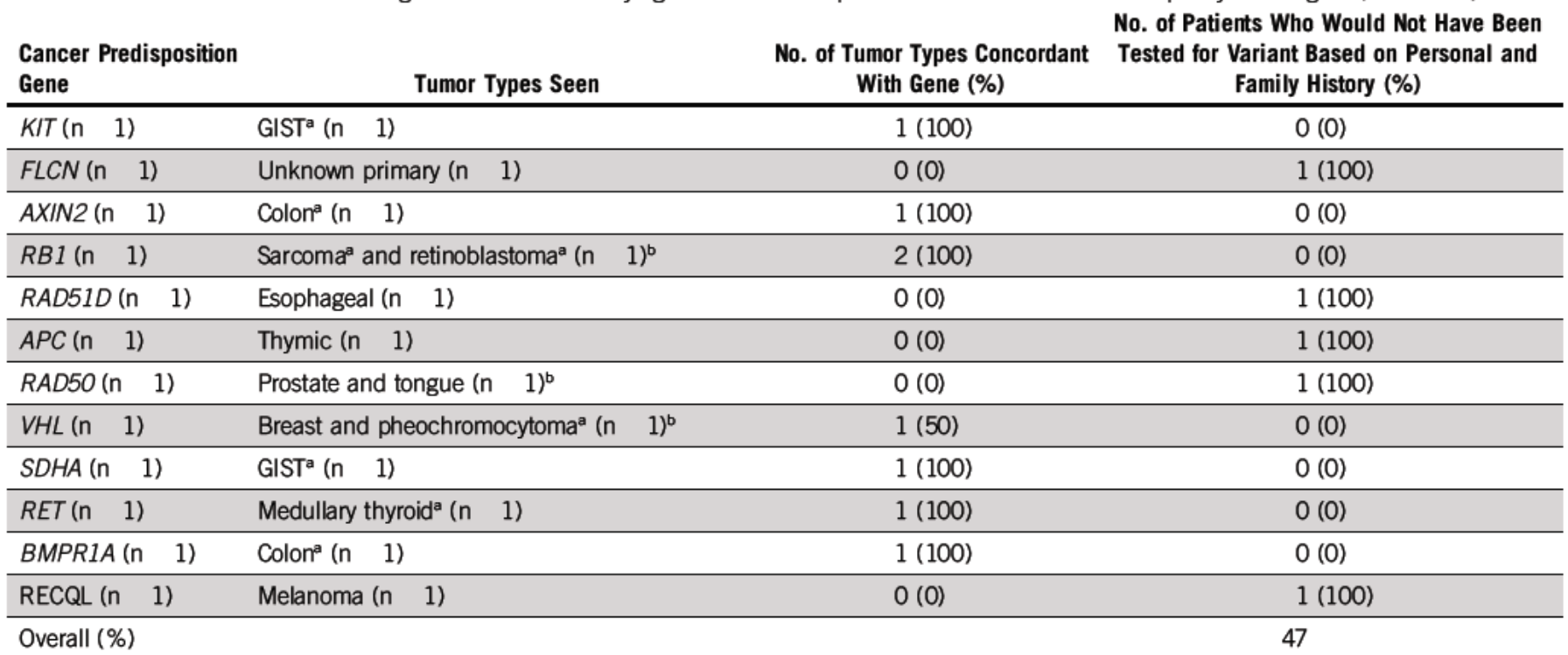

\footnotetext{
Abbreviations: ACC, adrenocortical carcinoma; GIST, GI stromal tumor; PNET, pancreatic neuroendocrine tumor; SCC, squamous cell carcinoma.

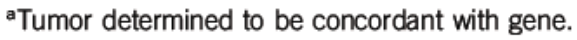

'Indicates patient had more than one primary malignancy.

${ }^{\mathrm{c}}$ Autosomal recessive condition; all identified patients in this category carried a single heterozygous mutation.
}

founder mutations known to be more common in that population.

\section{Frequency of ACMG-Recommended Actionable P/LP Variants}

The ACMG has assembled a list of 59 genes deemed to be clinically actionable and for which the results should be offered to patients undergoing clinical exome and genome analysis. ${ }^{17}$ Importantly, not all cancer predisposition variants are considered actionable, and not all actionable genes are cancer genes; the latter includes genes that can increase the risk of cardiac conditions, metabolic conditions, and others. The most commonly mutated ACMG genes with proposed management guidelines by the National Comprehensive Cancer Network (NCCN) and GeneReviews identified in our analysis were $A T P 7 B$ (2.7\%), BRCA2 (1.6\%), MUTYH (1.4\%), and BRCA1 ( $1 \%$; Table 4). After removing heterozygous ATP7B and MUTYH carriers, 69 patients $(6.7 \%)$ carried a mutation that would be recommended by the ACMG to be returned to the patient. In addition, $5.5 \%$ of patients $(4.2 \%$ of patients after removing MUTYH heterozygotes) carried an ACMG-designated, clinically actionable cancer P/LP gene variant.

\section{Relevance and Concordance of Pedigree With Cancer Risk Allele Carriage}

The electronic medical record of each patient carrying a P/LP variant in a cancer predisposition gene was reviewed by a licensed genetic counselor. Of 132 patients carrying a cancer predisposition variant, $51.9 \%$ had a three- generation pedigree with a focus on cancer history taken by a licensed genetic counselor. The family history for the remaining $48.1 \%$ of patients was taken from the clinic notes of the medical oncologist in the Precision Genomics clinic. As a result of the patients undergoing germline analysis, a thorough family history was obtained for each patient. On the basis of the patients' pertinent demographics and family history, we used current clinical practice guidelines ${ }^{18-20}$ to determine whether a patient would have been recommended to have genetic evaluation. If a patient's personal and family history would result in recommendation for genetic evaluation, a licensed genetic counselor determined which genetic test would likely have been offered to that patient using a multigene panel encompassing the tumor types identified in that patient's personal and family history. For patients who carried a cancer predisposing P/LP variant, $34 \%$ would not have been recommended to undergo testing, $13 \%$ would have been recommended to have a genetic test that would not have identified the uncovered P/LP variant, and $53 \%$ would have had the P/LP variant identified. For patients who carried an ACMG-recommended clinically actionable cancer predisposition P/LP variant, $23 \%$ would not have been recommended to undergo testing, $7 \%$ would have been recommended to have a genetic test that would not have identified the uncovered P/LP variant, and $70 \%$ would have had the P/LP variant identified. When considering the entire population, $6 \%$ and $1.7 \%$ of patients would not have had a cancer predisposition variant and an ACMGrecommended clinically actionable cancer predisposition variant, respectively, identified. 
TABLE 4. Patients With P/LP Variant in ACMG Recommended Gene and Associated Condition

ACMG-Recommended Gene

\begin{tabular}{ll}
\hline$A T P 7 B$ & Wilson disease ${ }^{\mathrm{a}, \mathrm{b}}$ \\
& \\
\hline BRCA2 & Hereditary breast and ovarian cancer
\end{tabular}

Inheritance

Pattern

AR Initiate treatment with copper chelating agents or zinc as soon as possible

At least twice annually: serum copper and ceruloplasmin, liver biochemistries, international normalized ratio, $\mathrm{CBC}$, urinalysis, and physical examination including neurologic assessment

At least once annually: 24 hour urinary excretion of copper

Women: clinical breast exam every 612 months starting at 25 years; annual MRI at 2575 years; annual mammogram at 3075 years; consider bilateral mastectomy; RRSO between age 35 and 40 years for $B R C A 1$ carriers but may be delayed until 4045 years for BRCA2; consider TVUS/CA 125 starting at 3035 years until RRSO

Men: clinical breast exam every 12 months starting at 35 years; prostate screening at 40 years

Women and men: no specific guidelines for melanoma screening; pancreatic cancer screening using MRI/MRCP and/or EUS may be considered based on family history

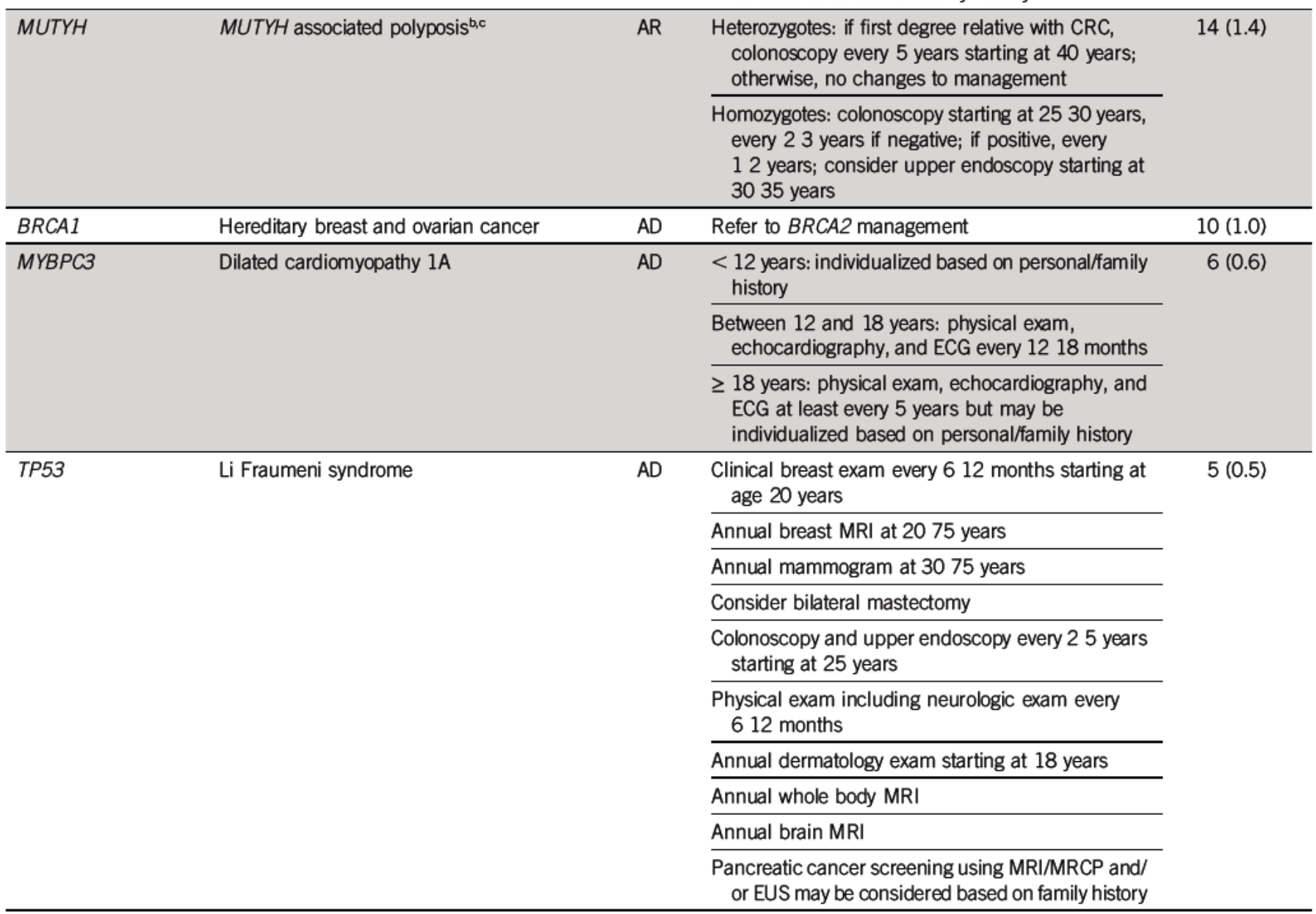

(Continued on following page)
No. of Patients

Carrying P/LP

Variant

( $n=1,028 ; \%)$

$28(2.7)$

$16(1.6)$ 
TABLE 4. Patients With P/LP Variant in ACMG Recommended Gene and Associated Condition (Continued)

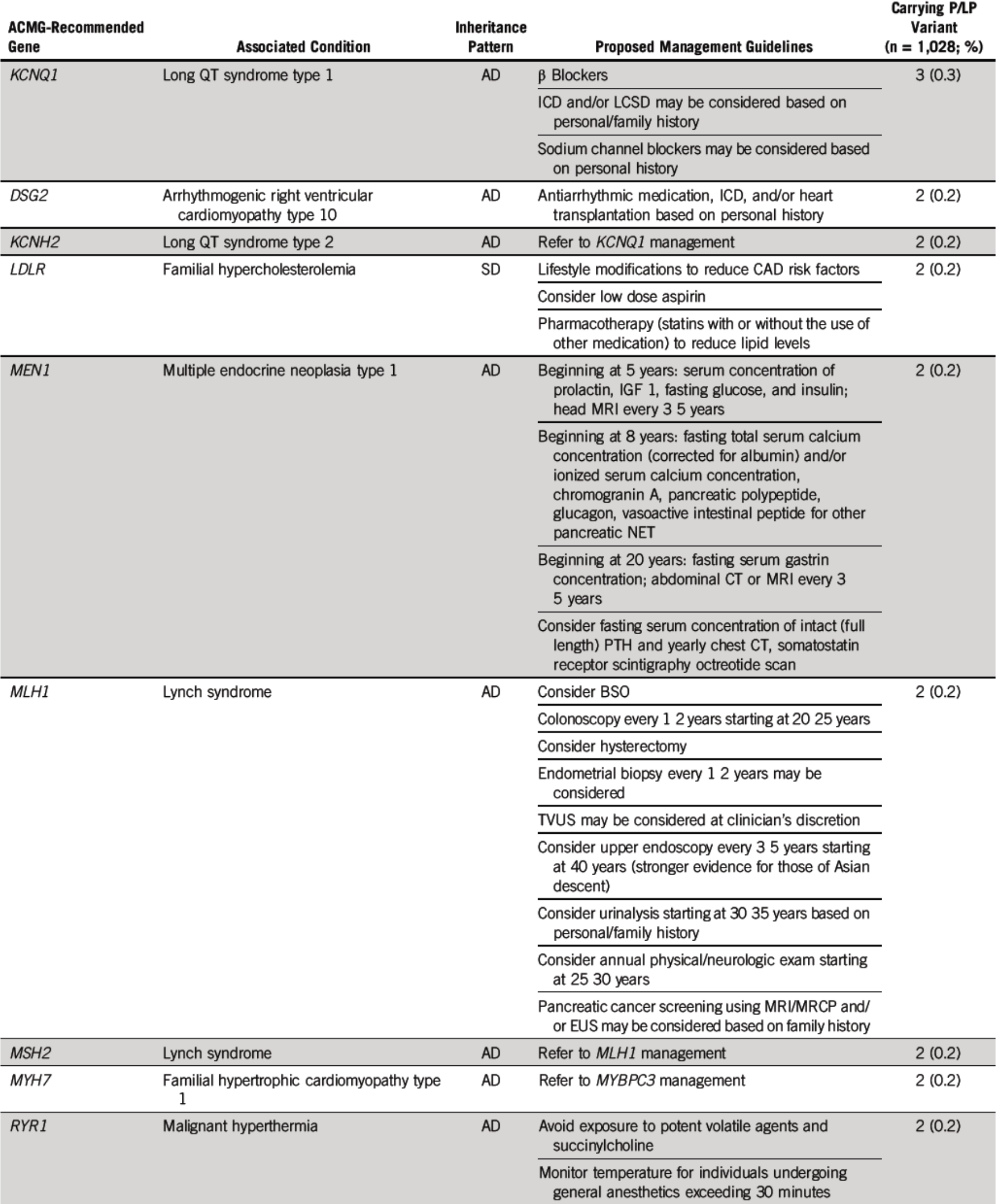


TABLE 4. Patients With P/LP Variant in ACMG Recommended Gene and Associated Condition (Continued)

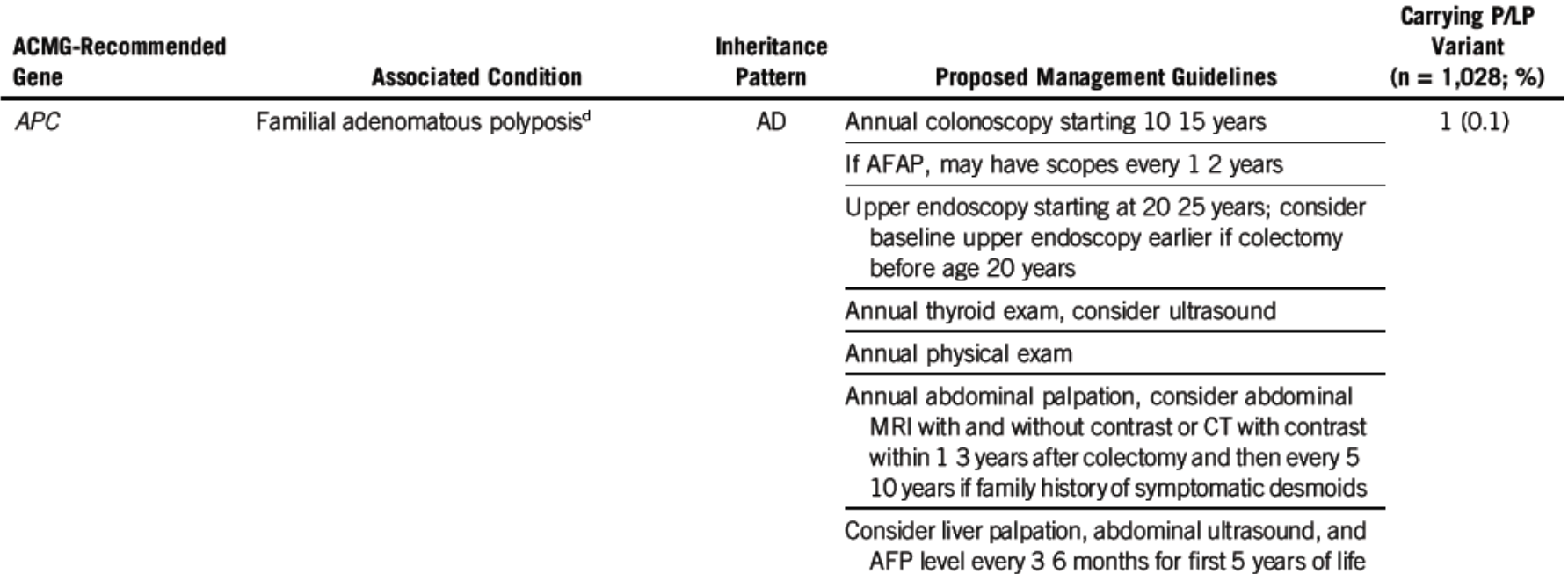

\begin{tabular}{llll}
\hline$A P O B$ & Familial hypercholesterolemia & SD & Refer to LDLR management \\
\hline BMPR1A & Juvenile polyposis syndrome & AD & $\begin{array}{c}\text { Beginning at } 15 \text { years: annual colonoscopy and } \\
\text { upper endoscopy; surveillance may be completed } \\
\text { every 2 3 years if no polyps are found }\end{array}$ \\
\hline
\end{tabular}

\begin{tabular}{|c|c|c|c|c|}
\hline DSC2 & $\begin{array}{l}\text { Arrhythmogenic right ventricular } \\
\text { cardiomyopathy type } 11\end{array}$ & $A D$ & Refer to DSG2 management & $1(0.1)$ \\
\hline \multirow[t]{2}{*}{ FBN1 } & Marfan syndrome & $A D$ & $\beta$ Blockers or angiotensin receptor blockers & $1(0.1)$ \\
\hline & & & $\begin{array}{l}\text { Annual echocardiography; frequency may be } \\
\text { increased based on personal history }\end{array}$ & \\
\hline
\end{tabular}

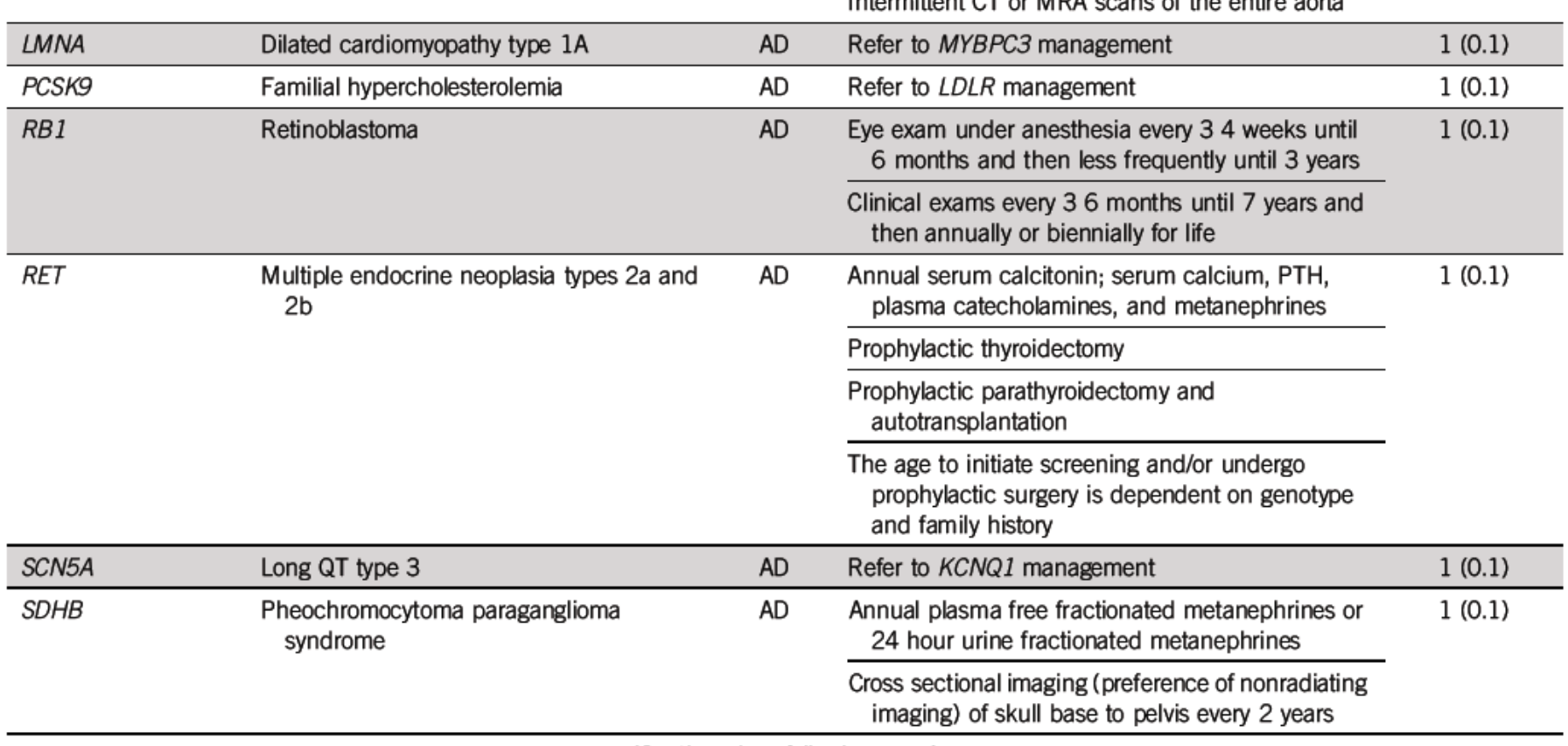


TABLE 4. Patients With P/LP Variant in ACMG Recommended Gene and Associated Condition (Continued)

\begin{tabular}{|c|c|c|c|c|}
\hline $\begin{array}{l}\text { ACMG-Recommended } \\
\text { Gene }\end{array}$ & Associated Condition & $\begin{array}{l}\text { Inheritance } \\
\text { Pattern }\end{array}$ & Proposed Management Guidelines & $\begin{array}{l}\text { Carrying P/LP } \\
\text { Variant } \\
(n=1,028 ; \%)\end{array}$ \\
\hline \multirow[t]{3}{*}{$V H L$} & Von Hippel Lindau syndrome & $A D$ & $\begin{array}{l}\text { Beginning at } 1 \text { year: ophthalmology exam, } \\
\text { evaluation for neurologic symptoms and hearing } \\
\text { loss, blood pressure monitoring (all completed } \\
\text { annually) }\end{array}$ & $1(0.1)$ \\
\hline & & & $\begin{array}{l}\text { Beginning at } 5 \text { years: annual plasma or } 24 \text { hour } \\
\text { urine for fractionated metanephrines; audiology } \\
\text { assessment every } 23 \text { years }\end{array}$ & \\
\hline & & & $\begin{array}{l}\text { Beginning at } 16 \text { years: annual abdominal } \\
\text { ultrasound; MRI of the brain, spine, and abdomen } \\
\text { every } 2 \text { years }\end{array}$ & \\
\hline
\end{tabular}

Abbreviations: ACMG, American College of Medical Genetics and Genomics; AD, autosomal dominant; AFAP, attenuated familial adenomatous polyposis; AFP, $\alpha$ fetoprotein; AR, autosomal recessive; BSO, bilateral salpingo oophorectomy; CAD, coronary artery disease; CRC, colorectal cancer; CT, computed tomography; EUS, endoscopic ultrasound; ICD, implantable cardioverter defibrillator; IGF 1, insulin like growth factor 1; LCSD, left cardiac sympathetic denervation; MRA, magnetic resonance angiography; MRCP, magnetic resonance cholangiopancreatography; MRI, magnetic resonance imaging; NET, neuroendocrine tumor; P/LP, pathogenic or likely pathogenic; PTH, parathyroid hormone; RRSO, risk reducing salpingo oophorectomy; SD, semidominant; TAH, total abdominal hysterectomy; TVUS, transvaginal ultrasound.

${ }^{a} A R$ condition; one patient in this category carried biallelic mutations.

${ }^{\mathrm{b}} \mathrm{ACMG}$ recommends disclosure of biallelic mutations only. Whole exome sequencing does not routinely include deletion and duplication analysis.

Additional genetic testing, additional screening for the condition, and/or thorough family history review may be considered to assess for a second germline mutation or moderately affected heterozygote carriers.

${ }^{\mathrm{C}} \mathrm{AR}$ condition; all identified patients in this category carried a single heterozygous mutation.

${ }^{\mathrm{d}}$ Patient has I1307K variant, which is associated with a modest risk for colon cancer, not considered familial adenomatous polyposis.

\section{DISCUSSION}

Tumor NGS has become an efficient approach for identifying actionable drug targets, and several studies have demonstrated benefit in outcomes for patients with advanced disease who receive this testing. ${ }^{21,22}$ Although the more comprehensive assessment of multiple targets increases efficiency of testing and potentially uncovers more drug options, it has also created the dilemma of triaging unexpected germline findings, which occur in $3.0 \%$ to $12.6 \%{ }^{4-9}$ of patients tested. This is further complicated by the fact that most patients do not have appropriate pretest counseling and that the purpose of the test is to identify a targeted agent, usually in the metastatic setting. ${ }^{23}$ These series all represent an assessment of germline variants initially identified on noncomprehensive panel-based testing, which does not account for all possible pathogenic variants and does not include paired germline testing. ${ }^{7}$ The former limitation likely underestimates the actual volume of germline carriers. The latter makes it impossible to know whether the finding is a reflection of a germline change. ${ }^{4}$ TCGA performed comprehensive WES on 10,389 patients and identified P/LP variants in $8 \% .{ }^{16}$ That study, however, focused entirely on cancer-relevant variants, and there were no clinical data to determine whether those patients should have been tested based on pedigree and other risk-specific factors. WES of tumor with matched normal sample in a small population of pediatric patients $(n=150)$ revealed that $10 \%$ had a germline P/LP mutation related to their disease and an additional $5 \%$ carried incidental germline mutations. ${ }^{8}$

To date, no prospective, comprehensive evaluation of cancer and noncancer germline variants with married clinical annotation has been reported for adult patients with cancer who are undergoing tumor NGS testing. We now report, to our knowledge, the first study of comprehensive germline WES with matched clinical annotation for adult patients with cancer undergoing testing with the intent of identifying a drug target. This comprehensive evaluation of the genetic landscape of this population highlights the pervasive nature of P/LP variants. The majority of the mutated genes in our data set with a frequency $>$ $1.5 \%$ were of autosomal recessive inheritance. In addition, only two $(6.9 \%)$ of 29 genes were recommended by the ACMG to be reported back to the patient. These findings highlight the pervasiveness of identifying mutations that are not clinically relevant to the patient and require expertise by the interpreting clinician. Nonetheless, $10.7 \%$ of patients did carry a P/LP variant in a cancer or a noncancer gene, which was frequently unexpected and would have been recommended by expert guidelines to be returned to the patient and acted on accordingly. Despite the relatively small numbers of ACMG reportable variants, pretest counseling should be performed to mitigate the downstream risks and harm of disclosing or not properly disclosing a potentially critical finding for both the patient and his or her family members. 
Although some of these mutation carriers may have been identified from germline screening prompted by risk, recent data from a subgroup of the IMPACT cohort highlighted concerns regarding the inability to adequately screen cancer mutation carriers based on pedigree. ${ }^{24}$ The IMPACT (ClinicalTrials.gov identifier: NCT01775022) cohort was a subcohort of 1,040 patients who were referred for additional germline testing, and $19.7 \%$ of patients were found to harbor a germline pathogenic variant. Astonishingly, approximately $50 \%$ of these patients would not have undergone germline testing based on their demographics, tumor characteristics, and pedigree and thus would have accounted for almost $10 \%$ of the population with a pathogenic variant who had not been recommended for testing. In this subcohort, patients were referred specifically for germline testing, and thus, although the pedigree did not always support formal testing, it does infer that the instinct of the treating physician is important. ${ }^{24}$ Furthermore, there was a high incidence of Ashkenazi Jewish founder pathogenic variants (27 of $59 B R C A$ variants and 24 of 24 APC variants) identified, suggesting a biased patient population that is already known to have a higher frequency of pathogenic germline variants in these genes. In our study, only $12.8 \%$ of patients harbored a germline cancer predisposition variant, and $5.5 \%$ had an ACMG cancer predisposition variant. Our study is a typical mix of all patients with cancer referred with no consideration of need for germline testing. The lower incidence of $P / L P$ variants in our study is likely in part a result of referral bias, tumor type, and patient population.

Despite the lower baseline rate of germline mutation carriage, we also found that between 30\% (ACMG cancer predisposition) and $47 \%$ (all cancer predisposition) of variants would not have been identified based on current clinical guidelines. We recognize the current clinical guidelines are designed to identify pathogenic variants in highly penetrant genes. Therefore, with some moderate penetrance genes, such as CHEK2 and ATM, it is expected that a higher number of pathogenic variants would be missed. Regardless, $1.7 \%$ of ACMG cancer predisposing variants, which are all in high-penetrance genes, would have been missed in a nonbiased referral population of patients with metastatic cancer intending to find a drug target. Although this represents a relatively small segment of the population, it is sobering that approximately $30 \%-50 \%$ of the patients with metastatic disease in this study harbored a risk allele that would not have been recommended for testing but may have led to risk-reducing screening or procedures had they been known earlier in the patient's life.

The clinical implications of these findings are complex and, based on these data, common. First, the setting itself imposes a unique challenge. Many patients in this setting have an incurable disease, and these findings were neither anticipated nor beneficial to the patient. Instead, the patient is now forced to consider the implications and burden of contacting other healthy family members who may be at risk. This may add an emotionally taxing burden that may also illicit unnecessary guilt and grief. ${ }^{25}$ Second, the findings themselves are mired in multiple considerations that are highly challenging for the medical oncologist to navigate.

Specifically, if a possible germline mutation is found on a traditional tumor-only panel-based test, there are no paired germline data and the oncologist is left to decide whether to pursue confirmatory testing. Currently, the NCCN guidelines ${ }^{18}$ formally recommend genetic evaluation for patients found to have a pathogenic variant in a cancer susceptibility gene while acknowledging the high frequency of somatic mutations clouding the picture for genes such as TP53. ${ }^{7,26}$ In addition, the impact of finding a cardiovascular or metabolic risk allele in a patient with end-stage cancer may seem unimportant and out of scope for the treating oncologist. However, there are data to suggest that many patients with metastatic cancer would prefer to receive these secondary findings. ${ }^{15,27}$

Other confounders exist for more comprehensive testing even though most have paired germline results. First, there are massive numbers of variants, and their pathogenicity designation is dynamic and best annotated by teams with extensive expertise in clinical and molecular genetics. ${ }^{28}$ Second, not all genes are clinically actionable, and thus, testing may only result in concern without offering an intervention to prevent or delay the onset. Third, even for clinically actionable genes, the penetrance is often variable, making the proposed benefit for family members to undergo testing less clear. ${ }^{29,30}$

The complexity of these findings underscores the concern regarding routine implementation of comprehensive NGS in clinical practice without ready access to expertise in variant interpretation and genetic counseling. This unintended need to consider germline findings places an increasing strain on time and resources for routine oncology practices as well as on the already limited number of genetic counselors. ${ }^{31}$ With the field quickly evolving, we foresee that all patients with advanced cancer who plan to undergo NGS for target drug identification should ideally have comprehensive pretest counseling so that the optimal results can be returned and expectations grounded. However, this optimal approach will strain the time and resources available in a routine oncology practice.

\section{AFFILIATIONS}

${ }^{1}$ Indiana University School of Medicine, Indianapolis, IN

${ }^{2}$ Indiana University Health Precision Genomics, Indianapolis, IN 


\section{CORRESPONDING AUTHOR}

Bryan P. Schneider, MD, Indiana University School of Medicine $1030 \mathrm{~W}$ Michigan St, Ste 3307, Indianapolis, IN 46202; e mail: bpschnei@ iu.edu.

\section{EQUAL CONTRIBUTION}

B.P.S. and L.A.S. contributed equally to this work.

\section{PRIOR PRESENTATION}

Presented at the 55th Annual Meeting of the American Society of Clinical Oncology, Chicago, IL, May 31 June 4, 2019.

\section{SUPPORT}

Supported by the Precision Health Initiative at Indiana University. The funders had no role in the design or execution of the study or the writing of the manuscript.

\section{AUTHOR CONTRIBUTIONS}

Conception and design: Bryan P. Schneider, Leigh Anne Stout, Patrick J. Kiel, Milan Radovich

Administrative support: Bryan P. Schneider, Milan Radovich

Provision of study material or patients: Bryan P. Schneider, Milan Radovich Collection and assembly of data: Bryan P. Schneider, Leigh Anne Stout, Courtney Schroeder, Nawal Kassem, Milan Radovich

Data analysis and interpretation: Bryan P. Schneider, Leigh Anne Stout, Santosh Philips, Courtney Schroeder, Susanna F. Scott, Cynthia Hunter Patrick J. Kiel, Milan Radovich
Manuscript writing: All authors

Final approval of manuscript: All authors

Accountable for all aspects of the work: All authors

\section{AUTHORS' DISCLOSURES OF POTENTIAL CONFLICTS OF INTEREST}

The following represents disclosure information provided by authors of this manuscript. All relationships are considered compensated unless otherwise noted. Relationships are self held unless noted. I = Immediate Family Member, Inst = My Institution. Relationships may not relate to the subject matter of this manuscript. For more information about ASCO's conflict of interest policy, please refer to www.asco.org/rwc or ascopubs. org/po/author center.

Open Payments is a public database containing information reported by companies about payments made to US licensed physicians (Open Payments).

\section{Patrick J. Kiel \\ Employment: Amgen \\ Speakers' Bureau: Takeda, Celgene, Genentech}

\section{Milan Radovich}

Stock and Other Ownership Interests: LifeOmic, Macrogenics, Immunomedics, ArQule, Tyme Technologies

Travel, Accommodations, Expenses: LifeOmic

No other potential conflicts of interest were reported.

\section{REFERENCES}

1. Blumenthal GM, Pazdur R: Approvals in 2017: Gene therapies and site agnostic indications. Nat Rev Clin Oncol 15:127 128, 2018

2. Freedman AN, Klabunde CN, Wiant K, et al: Use of next generation sequencing tests to guide cancer treatment: Results from a nationally representative survey of oncologists in the United States. JCO Precis Oncol 10.1200/PO.18.00169

3. van Nimwegen KJM, van Soest RA, Veltman JA, et al: Is the $\$ 1000$ genome as near as we think? A cost analysis of next generation sequencing. Clin Chem 62:1458 1464, 2016

4. Meric Bernstam F, Brusco L, Daniels M, et al: Incidental germline variants in 1000 advanced cancers on a prospective somatic genomic profiling protocol. Ann Oncol 27:795 800, 2016

5. Schrader KA, Cheng DT, Joseph V, et al: Germline variants in targeted tumor sequencing using matched normal DNA. JAMA Oncol 2:104 111, 2016

6. Seifert BA, O'Daniel JM, Amin K, et al: Germline analysis from tumor germline sequencing dyads to identify clinically actionable secondary findings. Clin Cancer Res 22:4087 4094, 2016

7. Jones S, Anagnostou V, Lytle K, et al: Personalized genomic analyses for cancer mutation discovery and interpretation. Sci Transl Med 7:283ra53, 2015

8. Parsons DW, Roy A, Yang Y, et al: Diagnostic yield of clinical tumor and germline whole exome sequencing for children with solid tumors. JAMA Oncol 2:616 624, 2016

9. Zhang J, Walsh MF, Wu G, et al: Germline mutations in predisposition genes in pediatric cancer. N Engl J Med 373:2336 2346, 2015

10. Yang S, Lincoln SE, Kobayashi Y, et al: Sources of discordance among germ line variant classifications in ClinVar. Genet Med 19:1118 1126, 2017

11. Gradishar W, Johnson K, Brown K, et al: Clinical variant classification: A comparison of public databases and a commercial testing laboratory. Oncologist 22:797 803, 2017

12. Balmaña J, Digiovanni L, Gaddam P, et al: Conflicting interpretation of genetic variants and cancer risk by commercial laboratories as assessed by the prospective registry of multiplex testing. J Clin Oncol 34:4071 4078, 2016

13. Robson ME, Bradbury AR, Arun B, et al: American Society of Clinical Oncology policy statement update: Genetic and genomic testing for cancer susceptibility. J Clin Oncol 33:3660 3667, 2015

14. Stjepanovic N, Stockley TL, Bedard PL, et al: Additional germline findings from a tumor profiling program. BMC Med Genomics 11:65, 2018

15. Yushak ML, Han G, Bouberhan S, et al: Patient preferences regarding incidental genomic findings discovered during tumor profiling. Cancer 122:1588 1597, 2016

16. Huang KL, Mashl RJ, Wu Y, et al: Pathogenic germline variants in 10,389 adult cancers. Cell 173:355 370.e14, 2018

17. Kalia SS, Adelman K, Bale SJ, et al: Recommendations for reporting of secondary findings in clinical exome and genome sequencing, 2016 update (ACMG SF v2.0): A policy statement of the American College of Medical Genetics and Genomics. Genet Med 19:249 255, 2017

18. National Comprehensive Cancer Network: Genetic/familial high risk assessment: Breast and ovarian version 3.2019. https://www2.tri kobe.org/nccn/guideline/ gynecological/english/genetic familial.pdf

19. National Comprehensive Cancer Network: Genetic/familial high risk assessment: Colorectal version 1.2018. https://www.nccn.org/about/news/ebulletin/ ebulletindetail.aspx?ebulletinid=294

20. Hampel H, Bennett RL, Buchanan A, et al: A practice guideline from the American College of Medical Genetics and Genomics and the National Society of Genetic Counselors: Referral indications for cancer predisposition assessment. Genet Med 17:70 87, 2015 
21. Radovich M, Kiel PJ, Nance SM, et al: Clinical benefit of a precision medicine based approach for guiding treatment of refractory cancers. Oncotarget 7:5649156500, 2016

22. Harada S, Arend R, Dai Q, et al: Implementation and utilization of the molecular tumor board to guide precision medicine. Oncotarget 8:57845 57854, 2017

23. Catenacci DVT, Amico AL, Nielsen SM, et al: Tumor genome analysis includes germline genome: Are we ready for surprises? Int J Cancer 136:1559 1567, 2015

24. Mandelker D, Zhang L, Kemel Y, et al: Mutation detection in patients with advanced cancer by universal sequencing of cancer related genes in tumor and normal DNA vs guideline based germline testing. JAMA 318:825 835, 2017

25. Biesecker BB: Genetic counselling: Psychological issues. In eLS, John Wiley \& Sons, Ltd (Ed.). doi:10.1002/9780470015902.a0005616.pub3

26. Weinstein JN, Collisson EA, Mills GB, et al: The Cancer Genome Atlas Pan Cancer analysis project. Nat Genet 45:1113 1120, 2013

27. Gray SW, Park ER, Najita J, et al: Oncologists' and cancer patients' views on whole exome sequencing and incidental findings: Results from the CanSeq study. Genet Med 18:1011 1019, 2016

28. Spurdle AB, Healey S, Devereau A, et al: ENIGMA Evidence based network for the interpretation of germline mutant alleles: An international initiative to evaluate risk and clinical significance associated with sequence variation in BRCA1 and BRCA2 genes. Hum Mutat 33:2 7, 2012

29. Katona BW, Yurgelun MB, Garber JE, et al: A counseling framework for moderate penetrance colorectal cancer susceptibility genes. Genet Med $20: 1324$ 1327, 2018

30. Tung N, Domchek SM, Stadler Z, et al: Counselling framework for moderate penetrance cancer susceptibility mutations. Nat Rev Clin Oncol 13:581 588, 2016

31. Hoskovec JM, Bennett RL, Carey ME, et al: Projecting the supply and demand for certified genetic counselors: A workforce study. J Genet Couns $27: 1620,2018$ 